\title{
Les territoires et les compétences collectives dans l'éducation au patrimoine en France
}

\author{
Territories and collective skills in heritage education in France
}

\author{
Sous la direction de : \\ Sylviane Blanc-Maximin ${ }^{1}$ et Angela Barthes ${ }^{2}$ \\ ${ }^{1}$ Aix-Marseille Université (France), Laboratoire ADEF, sylviane.maximin@univ-amu.fr \\ ${ }^{2}$ Aix-Marseille Université (France), Laboratoire ADEF, angela.barthes@univ-amu.fr
}

RÉSUMÉ. En France, les territoires sont de plus en plus porteurs de fonctions éducatives malgré une politique d'éducation toujours centralisée. L'éducation au patrimoine local constitue un exemple d'évolution des relations entre école et territoire mais questionne aussi les finalités éducatives formelles et non formelles, le fonctionnement des acteurs éducatifs et le potentiel des artefacts pour valoriser les ressources territoriales dans une dimension informelle. ABSTRACT. In France, territories are increasingly carrying educational functions despite a fairly centralized education policy.Local heritage education is an example of the evolution of the relationship between school and territory but also questions the formal and non-formal educational goals, the functioning of the actors and the potential of artifacts to enhance territorial resources in informal education.

MOTS-CLÉS. Territoires, éducation au patrimoine, compétences collectives, finalités éducatives.

KEYWORDS. Territories, heritage education, collective skills, educational goals.

Au-delà des textes législatifs convergents (Unesco, 1972, 2003), la notion de patrimoine reste ambiguë, le terme est ancien et a des acceptions changeantes selon l'époque et ses dépositaires. Audelà des apparences, sa définition n'est pas consensuelle, de même que l'identité reste un concept très largement débattu. La diversité des définitions reste néanmoins relative : toutes tendent à assimiler le patrimoine à une mémoire collective et, plus précisément, à une mémoire partagée propre à un groupe et à un territoire. Le patrimoine se réfère à l'expérience d'une communauté, avec l'idée qu'il s'ancre dans un passé et qu'il organise une capacité d'action. La mémoire collective est mobilisée pour affirmer la position de chacun et de tous dans le temps et dans la société, à ce titre elle ne peut pas être neutre. La notion de patrimoine est ainsi indissolublement liée à celle de pouvoir et la façon dont elle est constituée et définie, et donc appropriée par un groupe social, en constitue un enjeu de premier plan. Pour autant, la désignation officielle des patrimoines ne présume en rien de leur appropriation par les habitants et acteurs locaux, de la même manière que la désignation même d'un patrimoine ne va pas nécessairement de soi et s'appuie sur une construction sociale. Dans ce cadre, il est alors possible de souligner toute l'importance de la transmission des savoirs et des apprentissages qui sous-tendent les processus d'appropriation collectives des territoires dont les finalités seraient la mise en commun de valeurs partagées pour un groupe social.

A l'heure actuelle, ces apprentissages reposent sur l'idée d'une construction patrimoniale des territoires, lesquels peuvent être considérés comme des nouvelles ressources de développement. Les liens entre territoires, identités et patrimoine participent d'une approche économique du patrimoine. Mais celle-ci convoque l'éducation de manière de plus en plus prégnante dans les démarches de mise en patrimoine des territoires, ce qui conduit tout d'abord à interroger les rôles des territoires et des instances éducatives puis les interactions avec les dispositifs éducatifs formels et informels évolutifs et enfin, les processus éducatifs spécifiques à l'œuvre comme les " éducations à » (au développement durable, au patrimoine). Ce phénomène met au jour le double usage des formes 
d'éducations par les territoires et des territoires par l'École. A ce titre, il réinterroge les rôles de l'école et les formes scolaires.

Beaucoup de chercheurs se sont emparés des notions de patrimoine et de patrimonialisation, mais peu ont mis l'accent sur son accompagnement éducatif, comme l'un des piliers de leur construction. Pourtant il est aujourd'hui considéré comme fondamental, voire nécessaire à l'émergence patrimoniale, à tel point que l'éducation au patrimoine est rapidement devenue un objectif prioritaire au niveau mondial. En effet, L'article 27 de la convention du patrimoine mondial de l'UNESCO (1972) pose déjà les jalons du rôle important qui va être conféré à l'éducation en lien avec les construits patrimoniaux qu'elle formalise. Elle stipule en effet que « Les États parties à la présente Convention s'efforcent par tous les moyens appropriés, notamment par des programmes d'éducation et d'information, de renforcer le respect et l'attachement de leurs peuples au patrimoine culturel et naturel défini aux articles 1 et 2 de la Convention ». Le cadre supranational de l'éducation au patrimoine est posé puis repris et modifié dans les conventions concernant le patrimoine immatériel (2003), puis celle du Faro (2005)

Les quatre articles présentés dans ce numéro apportent des précisions sur les liens entre l'éducation au patrimoine et ses territoires. Angela Barthes, Serge Da Silva et Pierre Champollion s'attachent à montrer comment, historiquement, l'Éducation relative à l'environnement, l'Éducation au développement Durable et l'éducation au patrimoine local ont pris en compte l'objet territoire : comment ont été posés les jalons de la perspective du changement de paradigme des relations entre l'école et le territoire. Ils développent les grandes problématiques de l'éducation au patrimoine dans l'École aujourd'hui au niveau de la finalité éducative, de la légitimité des objets patrimoniaux à enseigner et de la place de ceux-ci en termes de contribution disciplinaire. Se posent alors les problèmes éthiques et épistémologiques avancés par Xavier Roth et Angela Barthes. Ils insistent alors sur le lien qui existe entre le problème éthique de la justification des «éducations à » dans lesquelles se mêlent savoirs et valeurs importées de la sphère sociopolitique et celles de la problématisation et finalités des éducations au patrimoine insérés dans leurs territoires. Ils montrent que des tensions sont à l'œuvre entre une éducation pensée comme entreprise de normalisation et présentant le risque d'un relativisme au niveau des savoirs enseignés. La problématisation prend appui sur une analyse des rapports entre savoirs et valeurs en histoire des sciences conduite par Canguilhem qui dépasse les écueils d'absolutisme et de relativisme à laquelle est également soumise l'éducation au patrimoine. Sylviane Blanc-Maximin, quant à elle s'intéresse aux partenariats d'éducation au patrimoine entre professeurs et intervenants d'un Parc Naturel Régional. La présence de nombreuses pratiques partenariales entre acteurs de l'éducation formelle (les enseignants) et non formelle (des intervenants extérieurs) pose des questions liées aux savoirs et aux valeurs enseignées. La focale ici choisie concerne le développement professionnel des acteurs éducatifs d'un territoire comprenant une aire protégée et pose la question de la mobilisation d'une compétence grâce à la rencontre de deux cultures professionnelles différentes et des conditions qui l'autorisent. Les notions de compétence d'un collectif et de compétence collective sont convoquées. Enfin Barbara Szafrajzen s'intéresse à la construction collective du savoir pour éduquer au patrimoine. L'auteure s'inscrit dans une épistémologie socio-constructiviste dans le cadre d'une éducation au patrimoine de personnes « séniors ». Elle contextualise cette éducation sur un territoire dans une structure muséale, qui propose une analyse sur une innovation numérique qui poursuit une double visée : faciliter l'accès au patrimoine local et permettre aux visiteurs de construire des savoirs dans le cadre d'une éducation informelle grâce à des artefacts. L'ensemble des contributions apportent des éclairages sur la complexité de l'éducation au patrimoine insérée dans une dimension territoriale et les compétences collectives qu'elle requiert 


\section{Bibliographie}

Barthes, A. Champollion, P. et Alpe, Y. (dir.). (2017). Permanences et évolutions de la relation complexes entre éducations et territoires, Série Education, ISTE Edition Ltd, Londres, 346 p.

Barthes A., Blanc-Maximin S. (2017). Quelles évolutions de l'école française face à l'éducation au patrimoine ? Revue des sciences de l'éducation. Vol 43. N¹. pp. 85-115. Montréal.

Blanc, S. et Eymard, C. (2010). Compétences d'un collectif d'école. Questions Vives, 5, 43-55.

Canguilhem, G. (2015). Euvres complètes, tome IV. Paris : Vrin.

Le Moigne, J-L. (2003). Le constructivisme. Modéliser pour comprendre. Tome 3. Paris : L’Harmattan.

Tutiaux-Guillon N. (2017). Education au patrimoine. In Barthes, Lange, et Tutiaux-Guillon (dir.). Dictionnaire critique des enjeux et concepts des éducations à. Paris : L’Harmattan. 